\title{
Analysis of variation in pre-procedural fasting duration for common inpatient gastrointestinal procedures
}

\author{
Vorada Sakulsaengprapha $^{1 \#}$, Michael Daniel ${ }^{2 \#}$, Jiarui Cai ${ }^{3}$, Diego A. Martinez ${ }^{3}$, Simon C. Mathews ${ }^{4,5}$ \\ ${ }^{1}$ Department of Medicine, Johns Hopkins University School of Medicine, Baltimore, MD, USA; ${ }^{2}$ Washington University School of Medicine in St. \\ Louis, Department of Gastroenterology, Barnes-Jewish Hospital, St. Louis, MO, USA; ${ }^{3}$ Division of Health Sciences Informatics, Department of \\ Emergency Medicine, Johns Hopkins University School of Medicine, Baltimore, MD, USA; ${ }^{4}$ Division of Gastroenterology and Hepatology, Johns \\ Hopkins Medicine, Baltimore, MD, USA; ${ }^{5}$ Johns Hopkins Medicine Armstrong Institute for Patient Safety and Quality, Baltimore, MD, USA \\ Contributions: (I) Conception and design: SC Mathews; (II) Administrative support: SC Mathews; (III) Provision of study materials or patients: SC \\ Mathews; (IV) Collection and assembly of data: J Cai, DA Martinez; (V) Data analysis and interpretation: J Cai, DA Martinez, SC Mathews, V \\ Sakulsaengprapha; (VI) Manuscript writing: All authors; (VII) Final approval of manuscript: All authors. \\ "These authors contributed equally to this work. \\ Correspondence to: Simon C. Mathews, MD. Division of Gastroenterology and Hepatology, Johns Hopkins Medicine, 600 North Wolfe Street, \\ Baltimore, MD 21287, USA. Email: smathe14@jhmi.edu.
}

Background: Gastrointestinal procedures generally require pre-procedural fasting to optimize sedation safety. While the American Society of Anesthesiologists (ASA) recommends no intake of clear liquids and solid food 2-4 and 6-8 hours respectively prior to endoscopic procedures, the actual nil per os (NPO) duration for these procedures in practice is unknown. Our objective was to analyze NPO duration for patients undergoing these procedures and to determine its association with clinical and administrative variables.

Methods: Inpatient data from 2016-2018 for the three procedures was extracted from electronic medical records and administrative data at a single-center tertiary academic medical center. Various statistical tests (Kruskal-Wallis, Wilcoxon, Pearson) were employed depending on the outcome type and data distribution.

Results: One thousand three hundred and twenty-five esophagogastroduodenoscopies (EGDs), 753 colonoscopies, and 550 endoscopic retrograde cholangiopancreatographies (ERCPs) were included. The median NPO time for all procedures was 12.6 hours (IQR, 9.6-16.1 hours). The median NPO times were 12.6, 11.9, and 13.1 hours for EGD, colonoscopy, and ERCP respectively. NPO duration was greater for Hispanic than non-Hispanic patients (median 13.9 vs. 12.4, $\mathrm{P}=0.018$ ). NPO duration was also associated with increased age $(r=0.041, \mathrm{P}=0.027)$ and inversely related to hospital occupancy $(\mathrm{r}=-0.08, \mathrm{P}<0.0001)$. There were no statistically significant associations with provider type, hospital location or service, length of stay, and total number of comorbidities.

Conclusions: NPO times for common inpatient gastroenterology (GI) procedures generally exceeded 12 hours, suggesting there is an opportunity to adopt changes to decrease NPO duration for low-risk patients while maintaining adherence to guidelines and best practice.

Keywords: Endoscopy; patient experience; fasting

Received: 02 August 2020; Accepted: 21 January 2021; Published: 25 October 2022.

doi: $10.21037 /$ tgh-20-280

View this article at: http://dx.doi.org/10.21037/tgh-20-280 


\section{Introduction}

Gastrointestinal procedures are common with an estimated 11.0 million colonoscopies, 6.1 million esophagogastroduodenoscopies (EGDs), and 169,500 endoscopic retrograde cholangiopancreatographies (ERCPs) performed each year (1). Many of these procedures occur in the inpatient setting and generally include some form of sedation, which can impair the upper airway protective reflexes, increasing the perioperative risk of regurgitation and pulmonary aspiration (2). As a result, patients are generally required to fast pre-procedurally to minimize the risks associated with sedation unless in case of emergency. However, fasting can be associated with decreased patient dissatisfaction (3) and can potentially impact clinical outcomes $(4,5)$ through mechanisms such as dehydration and hypoglycemia (6).

Although there is known variation in inpatient nil per os (NPO) practices (6), guidelines from the American Society of Anesthesiologists (ASA) recommend no intake of clear liquids and solid food 2-4 and 6 hours (8 hours if intake includes meat, fried or fatty foods) respectively prior to endoscopic procedures (2). The ASA cites randomized control trials which demonstrate less thirst and hunger as well as lower risk of aspiration for the recommended clear liquid guidelines compared to higher fasting time (over 4 hours) (7-9). These studies also found no difference in terms of gastric volume, gastric $\mathrm{pH}$, and blood glucose values when comparing 2-4 hours and greater than 4 hours nutritional or carbohydrate drink fasting (10-13). Moreover, it has been found that a 6- and 1-hour fasting period for solids and water respectively prior to upper gastrointestinal (GI) endoscopy yields good endoscopic vision as well as minimizes patient discomfort (14).

However, despite the evidence in favor of short preprocedural fasting durations, clinical practice patterns at hospitals around the world commonly begin NPO orders at midnight prior to a procedure regardless of the scheduled time of the procedure (3). This scenario may be exacerbated when there is ambiguity in diagnostic or treatment plans resulting in patients being left NPO for days. Because long inpatient pre-procedural fasting can negatively impact a patient's experience as well as clinical outcomes, the objective of our study was to understand better what typical NPO patterns for common GI procedures are and how they might vary by demographic, clinical, and operational characteristics at a large academic medical center. We present the following article in accordance with the MDAR reporting checklist (available at https://tgh. amegroups.com/article/view/10.21037/tgh-20-280/rc).

\section{Methods}

The study was conducted by The Johns Hopkins Hospital Office of Patient Experience and the Division of Gastroenterology using de-identified data for the purposes of quality improvement. The study conformed to the provisions of the Declaration of Helsinki (as revised in 2013). Data for this study was extracted retrospectively from the Casemix data system, and the Epic electronic medical record. Adult patients (age 18 or older) included in this analysis had either an EGD, colonoscopy, or ERCP associated with their inpatient hospital stay, who were admitted between July 28, 2016 and January 31, 2018 (553 days duration). Exclusion criteria include NPO times over 72 hours, as these patients likely had other reasons for their NPO status. This threshold was determined through a general survey of inpatient clinical providers.

NPO times were calculated based on the duration of time between when the NPO order went into effect and when the "Patient in Procedure Room" order was entered. If multiple NPO orders were placed on the same patient during a single hospitalization, instances included for the study were the NPO order that went into effect most proximally prior to the aforementioned procedures.

\section{Statistical analysis}

The timing, duration, and number of NPO orders in relation to their procedures were tallied. Statistical analysis was performed using the $\mathrm{R}$ statistical package. KruskalWallis tests, Wilcoxon tests, and Pearson tests were used depending on the outcome type and data distribution. In comparing the distribution of two groups, the Wilcoxon statistical test was used. For comparing more than two groups, the Kruskal-Wallis test was used. Lastly, the Pearson test was used to assess linear correlation.

\section{Results}

During the study period, 2,913 hospitalizations and ED visits at the Johns Hopkins Hospital with at least one associated colonoscopy [796], EGD [1,526] or ERCP [591] procedure were included. Most patients were male (51\%), white (53\%), non-Hispanics $(96 \%)$ with an average and median age of 60 years, (Q25\%: 47 years and Q75\%: 69 years). 


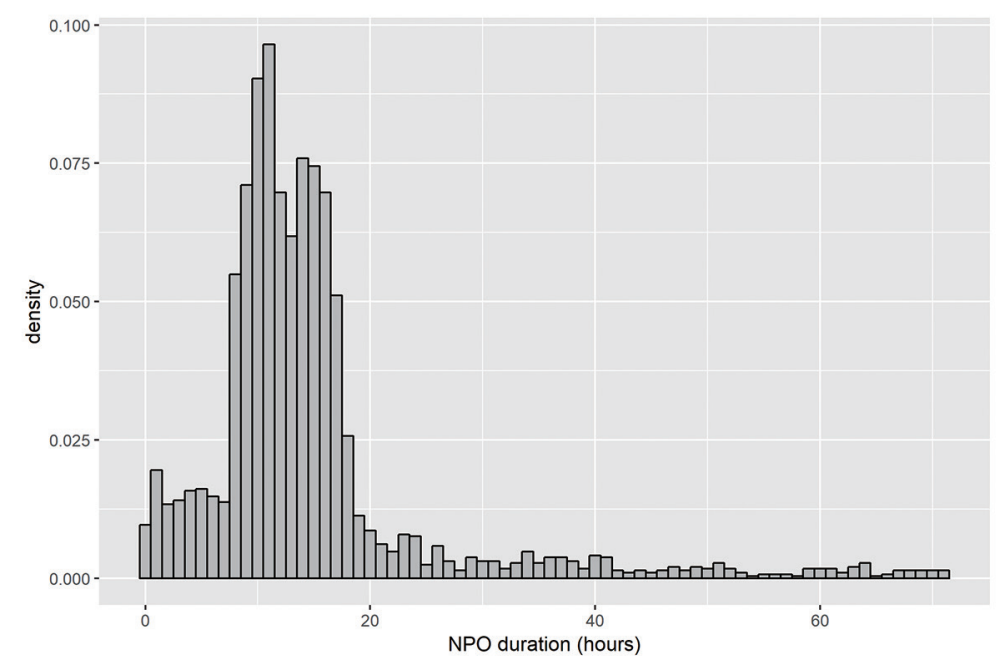

Figure 1 Distribution of NPO wait times (median 12.6 hours). NPO, nil per os.

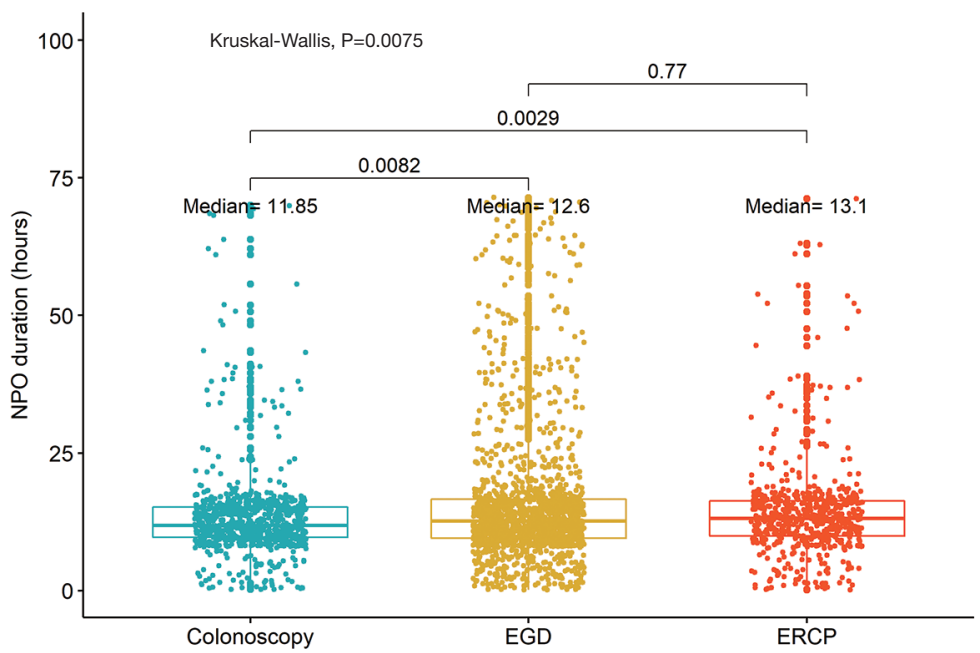

Figure 2 Distribution of NPO wait times by procedure: colonoscopy (median 11.9 hours), EGD (median 12.6 hours), and ERCP (median 13.1 hours). NPO, nil per os; EGD, esophagogastroduodenoscopy; ERCP, endoscopic retrograde cholangiopancreatography.

The median duration of patient NPO status for all procedures was 12.60 hours (IQR, 9.60-16.10 hours; Figure 1). The median NPO duration for ERCPs was 13.1 hours, while the median NPO durations for EGDs and colonoscopies were 12.6 and 11.9 hours, respectively (Figure 2). NPO durations for EGDs and ERCPs were statistically greater than those for colonoscopies ( $\mathrm{P}=0.0082$ and 0.0029$)$.

The distribution of times the NPO orders went into effect are depicted in Figure 3 with approximately $63 \%$ of orders effective at midnight. The remainder of NPO orders were largely evenly distributed throughout the day.
Duration of NPO was stratified by race and ethnicity. The median NPO time for Black/African-American patients was 11.9 hours; 12.6 hours for White/Caucasian patients; and for "other" patients was 13.8 hours. The difference between the "other" category and the former categories was statistically significant $(\mathrm{P}=0.0037$ and 0.013, respectively; Figure 4). Asian patients did not have statistically significantly different NPO times compared to other groups. Hispanic patients spent more time NPO than non-Hispanic patients (13.9 vs. 12.4 hours, respectively, $\mathrm{P}=0.018$ ). Differences were also seen based on age, with 


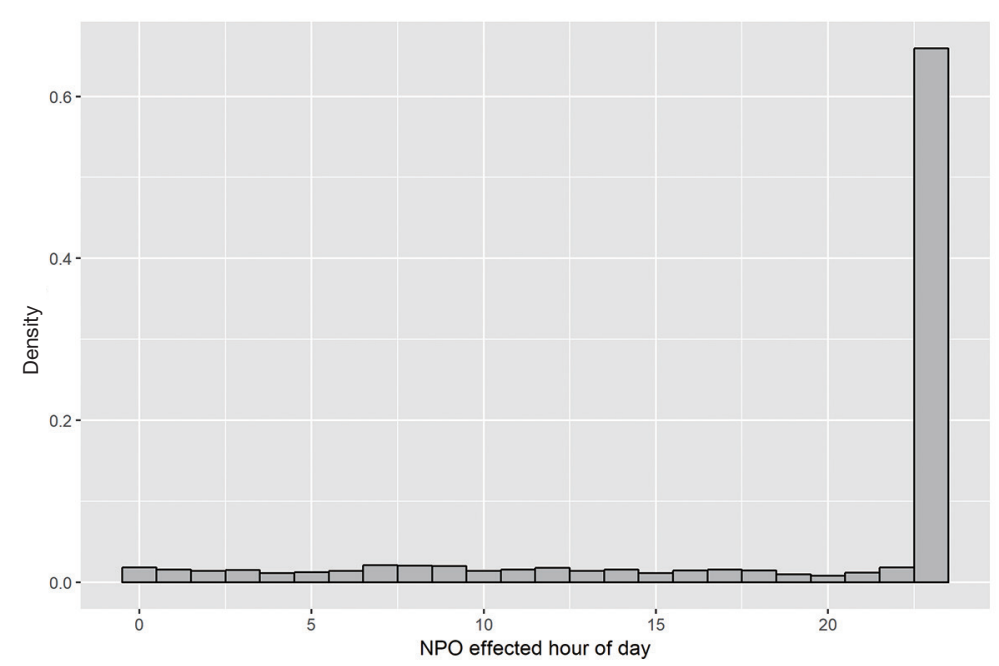

Figure 3 Distribution of times throughout the day for when NPO orders went into effect. NPO, nil per os.

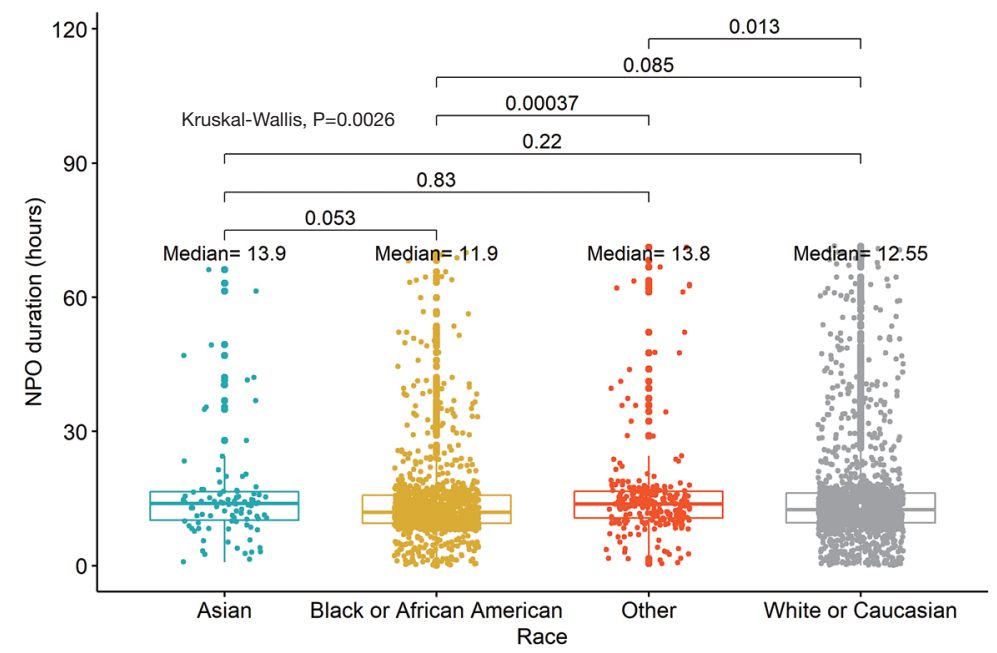

Figure 4 Distribution of NPO times by race: Asian (median 13.9 hours), Black/African American (median 11.9 hours), other (median 13.8 hours), and White/Caucasian (median 12.55 hours). NPO, nil per os.

increasing age correlating with longer NPO times $(\mathrm{r}=0.041$, $\mathrm{P}=0.027$; Figure 5).

There was a statistically significant negative correlation between inpatient hospital occupancy and NPO times, indicating that patients were found to spend less time $\mathrm{NPO}$ as inpatient hospital occupancy increased $(\mathrm{r}=-0.08$, $\mathrm{P}<0.0001$; Figure 6). There was no statistical difference in NPO duration across the 53 units and 26 provider teams included in the cohort (Figure 7). In addition, there was no statistically significant association between NPO duration and: patients' sex; number of comorbidities; intensive care unit days; length of stay; or provider type (physician or nonphysician).

\section{Discussion}

The vast majority of the three common GI procedures had NPO durations between 10 and 16 hours as represented by the $25-75 \%$ interquartile range. These values may largely be explained by the historical practice of keeping patients NPO after midnight. However, with ASA guidelines recommending a clear liquids fast duration between $2-4$ hours and increasing 


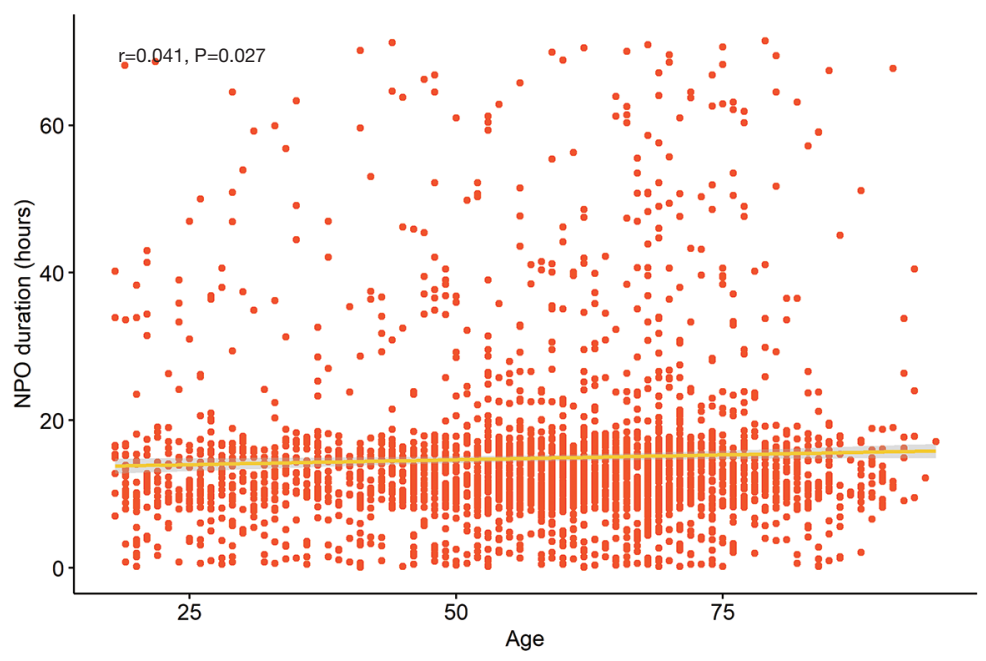

Figure 5 Association of NPO times and age. Greater age is correlated with increased NPO duration. NPO, nil per os.

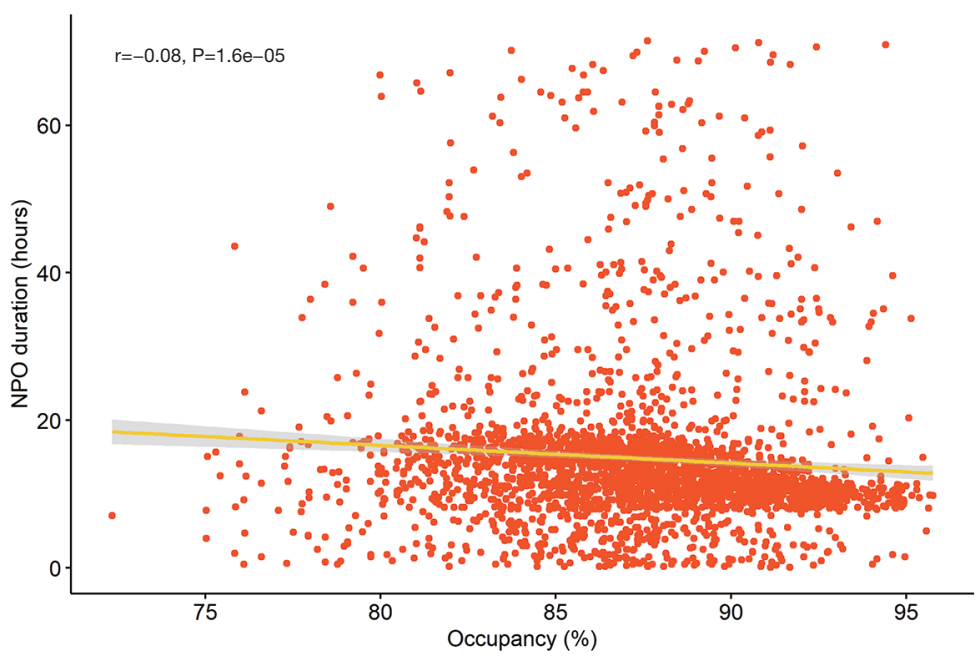

Figure 6 Association of NPO times and hospital bed occupancy. NPO duration decreases with increasing hospital occupancy. NPO, nil per os.

evidence to support the benefits of decreased fasting durations (2), there is likely an opportunity to improve the current clinical practice at our institution. Liberalizing NPO durations will not be appropriate for all patients, particularly those that are acutely ill, have unknown trajectories, or have established co-morbid conditions that confer increased risk. However, given the majority of patients undergoing inpatient procedures are not in extremis, identifying lowerrisk patients who can tolerate a decreased NPO durations, may improve overall experience for these patients without sacrificing clinical outcomes which has been in supported in other clinical contexts (15-17). The utilization of new digital tools, such as electronic decision support, may aid in streamlining the process of risk-stratifying patients. This has been demonstrated successfully in other clinical areas, such as deep vein thrombosis prophylaxis (18). Another possible intervention, given that providers commonly prescribe NPO after midnight regardless of scheduled procedure time, would be to implement an NPO prescribing algorithm within the electronic health record that calculates the NPO start time based on scheduled procedure time rather than making the NPO order effective at a particular time irrespective of the 


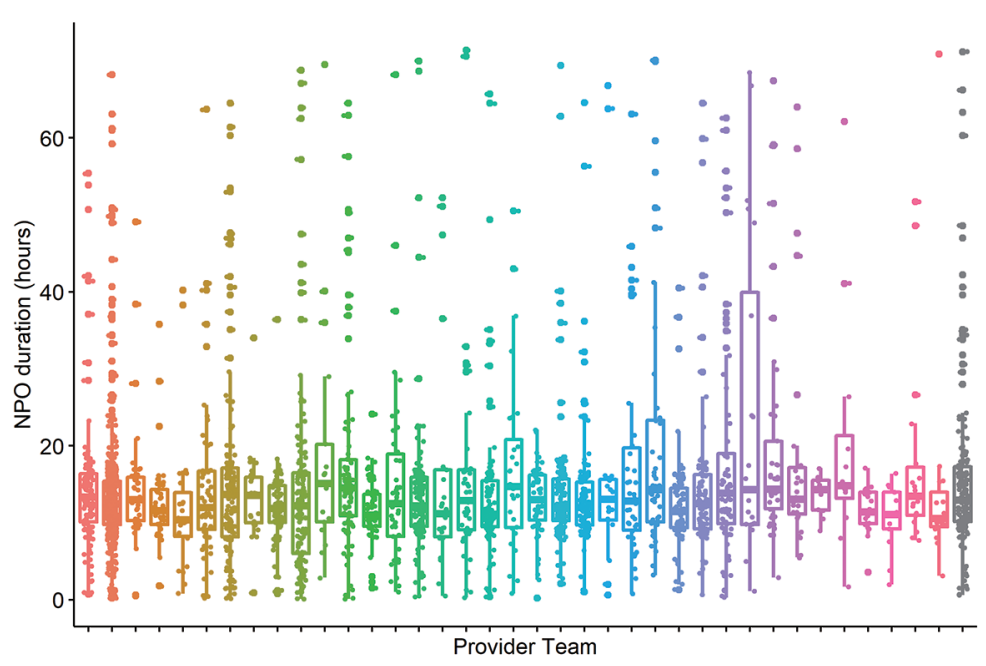

Figure 7 Distribution of NPO time provider team with each boxplot representing unique teams. No statistical differences were found across provider teams. NPO, nil per os.

patient's schedule.

The differences in NPO duration between Hispanic and non-Hispanic patients may be a proxy for the impact of potential language barriers resulting in care delays which has been previously established $(19,20)$. However, since language proficiency and other variables such as nursing staffing ratio and communication modality were not available, this relationship cannot be established. The relationship between increasing age and longer NPO duration may also be a manifestation of established disparities described in the care of the elderly $(21,22)$, though the absolute differences in times across the age spectrum are still relatively small. The inverse relationship with hospital occupancy may reflect a heightened urgency to get patients treated sooner to reduce a backlog of cases. The lack of correlation with length of stay suggests that these GI procedures may not have been the rate-limiting step in a patient's discharge. In addition, the lack of variation across provider teams and hospital locations, suggest that NPO duration is relatively insulated from the nuances based on clinical specialty or local geographic practice.

\section{Limitations}

The data for this study was collected retrospectively which limits any inferences on causality of NPO times. However, the focus of this analysis was to characterize the nature of NPO duration and to understand the variation in practice. In-depth case review was not feasible in this context. Future investigation should include the prospective collection of clinical variables, including indication for procedure, urgency of procedure, active clinical co-morbidities, and pre- and post-laboratory values for hypovolemia and hypoglycemia. In addition, operational data such as nursing staffing ratios, time since GI consultation, time of EHR order entry, and characterization of procedure delays would provide understanding into the causal relationships impacting fasting duration.

Lastly, the study being performed at a single institution may also be a limitation to generalizability. However, this is mitigated by the fact that the sample size included in this study was large and reflected a diverse community. In addition, inpatient endoscopy workflow protocols are typically similar across institutions.

\section{Significance}

Our findings are the first to describe inpatient NPO durations for common GI procedures and explore variations across these times. With its large, diverse sample of procedures, our study findings are likely extrapolatable to other large academic medical centers and perhaps even more broadly to all hospitalized patients undergoing these procedures given that endoscopy workflow is generalizable.

\section{Conclusions}

NPO times for common inpatient GI procedures are 
commonly over 12 hours in duration. These findings may provide the needed context to support future changes to how NPO times are implemented in practice. With this, there is a clear opportunity to adopt changes in practice to decrease NPO duration for low-risk patients and still maintain adherence to guidelines and best practice. Future studies should further evaluate the impact of NPO duration on clinical outcomes as well as quality metrics such as patient experience scores. In addition, the underlying drivers of disparities in NPO times should also be explored.

\section{Acknowledgments}

Funding: None.

\section{Footnote}

Reporting Checklist: The authors have completed the MDAR reporting checklist. Available at https://tgh.amegroups.com/ article/view/10.21037/tgh-20-280/rc

Conflicts of Interest: All authors have completed the ICMJE uniform disclosure form (available at https://tgh.amegroups. com/article/view/10.21037/tgh-20-280/coif). The authors have no conflicts of interest to declare.

Ethical Statement: The authors are accountable for all aspects of the work in ensuring that questions related to the accuracy or integrity of any part of the work are appropriately investigated and resolved. The study conformed to the provisions of the Declaration of Helsinki (as revised in 2013).

Open Access Statement: This is an Open Access article distributed in accordance with the Creative Commons Attribution-NonCommercial-NoDerivs 4.0 International License (CC BY-NC-ND 4.0), which permits the noncommercial replication and distribution of the article with the strict proviso that no changes or edits are made and the original work is properly cited (including links to both the formal publication through the relevant DOI and the license). See: https://creativecommons.org/licenses/by-nc-nd/4.0/.

\section{References}

1. Peery AF, Crockett SD, Murphy CC, et al. Burden and Cost of Gastrointestinal, Liver, and Pancreatic Diseases in the United States: Update 2018. Gastroenterology 2019;156:254-72.e11.

2. Practice guidelines for preoperative fasting and the use of pharmacologic agents to reduce the risk of pulmonary aspiration: application to healthy patients undergoing elective procedures: an updated report by the American Society of Anesthesiologists Task Force on preoperative fasting and the use of pharmacologic agents to reduce the risk of pulmonary aspiration. Anesthesiology 2017;126:376-93.

3. Chon T, Ma A, Mun-Price C. Perioperative fasting and the patient experience. Cureus 2017;9:e1272.

4. Marik PE, Zaloga GP. Early enteral nutrition in acutely ill patients: a systematic review. Crit Care Med 2001;29:2264-70.

5. Doig GS, Heighes PT, Simpson F, et al. Early enteral nutrition, provided within $24 \mathrm{~h}$ of injury or intensive care unit admission, significantly reduces mortality in critically ill patients: a meta-analysis of randomised controlled trials. Intensive Care Med 2009;35:2018-27.

6. Sorita A, Thongprayoon C, Ratelle JT, et al. Characteristics and outcomes of fasting orders among medical inpatients. J Hosp Med 2017;12:36-9.

7. Agarwal A, Chari P, Singh H. Fluid deprivation before operation. The effect of a small drink. Anaesthesia 1989;44:632-4.

8. Itou K, Fukuyama T, Sasabuchi Y, et al. Safety and efficacy of oral rehydration therapy until $2 \mathrm{~h}$ before surgery: a multicenter randomized controlled trial. J Anesth 2012;26:20-7.

9. Phillips S, Hutchinson S, Davidson T. Preoperative drinking does not affect gastric contents. Br J Anaesth 1993;70:6-9.

10. Yagci G, Can MF, Ozturk E, et al. Effects of preoperative carbohydrate loading on glucose metabolism and gastric contents in patients undergoing moderate surgery: a randomized, controlled trial. Nutrition 2008;24:212-6.

11. Wang ZG, Wang Q, Wang WJ, et al. Randomized clinical trial to compare the effects of preoperative oral carbohydrate versus placebo on insulin resistance after colorectal surgery. Br J Surg 2010;97:317-27.

12. Bopp C, Hofer S, Klein A, et al. A liberal preoperative fasting regimen improves patient comfort and satisfaction with anesthesia care in day-stay minor surgery. Minerva Anestesiol 2011;77:680-6.

13. Yildiz H, Gunal SE, Yilmaz G, et al. Oral carbohydrate supplementation reduces preoperative discomfort in laparoscopic cholecystectomy. J Invest Surg 2013;26:89-95. 
14. De Silva AP, Amarasiri L, Liyanage MN, et al. Onehour fast for water and six-hour fast for solids prior to endoscopy provides good endoscopic vision and results in minimum patient discomfort. J Gastroenterol Hepatol 2009;24:1095-7.

15. Crenshaw JT. Preoperative fasting: will the evidence ever be put into practice? Am J Nurs 2011;111:38-43.

16. Shaukat A, Malhotra A, Greer N, et al. Systematic review: outcomes by duration of NPO status prior to colonoscopy. Gastroenterol Res Pract 2017;2017:3914942.

17. Shah JN, Maharjan S, Gurung R. Shortened preoperative fasting time to allow oral rehydration solution clear liquid up to two hours before elective major surgery in adults. J Coll Physicians Surg Pak 2018;28:348-51.

18. Streiff MB, Carolan HT, Hobson DB, et al. Lessons from the johns hopkins multi-disciplinary venous thromboembolism (VTE) prevention collaborative. BMJ

doi: 10.21037/tgh-20-280

Cite this article as: Sakulsaengprapha V, Daniel M, Cai J, Martinez DA, Mathews SC. Analysis of variation in pre-procedural fasting duration for common inpatient gastrointestinal procedures. Transl Gastroenterol Hepatol 2022;7:39. 2012;344:e3935.

19. Garcia CM, Duckett LJ. No te entiendo y tú no me entiendes: language barriers among immigrant Latino adolescents seeking health care. J Cult Divers 2009;16:120-6.

20. Calo WA, Cubillos L, Breen J, et al. Experiences of Latinos with limited English proficiency with patient registration systems and their interactions with clinic front office staff: an exploratory study to inform communitybased translational research in North Carolina. BMC Health Serv Res 2015;15:570.

21. Salway SM, Payne N, Rimmer M, et al. Identifying inequitable healthcare in older people: systematic review of current research practice. Int J Equity Health 2017;16:123.

22. Yamada T, Chen CC, Murata C, et al. Access disparity and health inequality of the elderly: unmet needs and delayed healthcare. Int J Environ Res Public Health 2015;12:1745-72. 